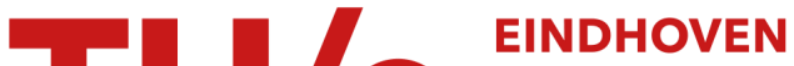 UNIVERSITY OF TECHNOLOGY
}

\section{Context weighting for general finite context sources}

Citation for published version (APA):

Willems, F. M. J., Shtarkov, Y. M., \& Tjalkens, T. J. (1996). Context weighting for general finite context sources. IEEE Transactions on Information Theory, 42(5), 1514-1520. https://doi.org/10.1109/18.532891

DOI:

10.1109/18.532891

Document status and date:

Published: 01/01/1996

\section{Document Version:}

Publisher's PDF, also known as Version of Record (includes final page, issue and volume numbers)

\section{Please check the document version of this publication:}

- A submitted manuscript is the version of the article upon submission and before peer-review. There can be important differences between the submitted version and the official published version of record. People interested in the research are advised to contact the author for the final version of the publication, or visit the $\mathrm{DOI}$ to the publisher's website.

- The final author version and the galley proof are versions of the publication after peer review.

- The final published version features the final layout of the paper including the volume, issue and page numbers.

Link to publication

\section{General rights}

Copyright and moral rights for the publications made accessible in the public portal are retained by the authors and/or other copyright owners and it is a condition of accessing publications that users recognise and abide by the legal requirements associated with these rights.

- Users may download and print one copy of any publication from the public portal for the purpose of private study or research.

- You may not further distribute the material or use it for any profit-making activity or commercial gain

- You may freely distribute the URL identifying the publication in the public portal.

If the publication is distributed under the terms of Article $25 f a$ of the Dutch Copyright Act, indicated by the "Taverne" license above, please follow below link for the End User Agreement:

www.tue.nl/taverne

Take down policy

If you believe that this document breaches copyright please contact us at:

openaccess@tue.nl

providing details and we will investigate your claim. 


\section{Correspondence}

\section{Context Weighting for General Finite-Context Sources}

Frans M. J. Willems, Member, IEEE, Yuri M. Shtarkov, and Tjalling J. Tjalkens, Member, IEEE

\begin{abstract}
C Context weighting procedures are presented for sources with models (structures) in four different classes. Although the procedures are designed for universal data compression purposes, their generality allows application in the area of classification.
\end{abstract}

Index Terms-Sequential data compression, universal source coding, finite-context sources, modeling procedure, arithmetic coding, cumulative redundancy bounds, classification procedure.

\section{INTRODUCTION}

Recently in [13], [14] the authors introduced context-tree weighting as a sequential universal source-coding method for the class of binary (bounded memory) tree sources. Tree sources were defined around the same time by Weinberger et al. [12]. The idea behind context weighting procedures can be summarized as follows: ${ }^{1}$

The well-known Elias algorithm (described in, e.g., Jelinek [1]) produces for any coding distribution $P_{c}\left(x_{1}^{T}\right)$ over all binary sequences of length $T$, a binary prefix code with codeword lengths $L\left(x_{1}^{T}\right)$ that satisfy

$$
L\left(x_{1}^{T}\right)<\log \frac{1}{P_{c}\left(x_{1}^{T}\right)}+2, \text { for all } x_{1}^{T} .
$$

(We assume that the base of the $\log (\cdot)$ is 2 . Codeword lengths and information quantities are expressed in bits.) If the marginals of the coding distribution

$$
P_{c}\left(x_{1}^{t}\right)=\sum_{x_{t+1}^{T}} P_{c}\left(x_{1}^{T}\right), \quad \text { for } t=1, \cdots, T
$$

are sequentially available, the Elias (arithmetic) code can be implemented sequentially. Accepting a coding redundancy of at most 2 bits, we are now left with the problem of finding good coding distributions $P_{c}(\cdot)$.

For binary memoryless sources with an unknown parameter $\theta$ (i.e., the probability of generating a 1 ), it is reasonable to assign the block probability $P_{c}\left(x_{1}^{T}\right)=P_{e}(a, b)$ to a sequence $x_{1}^{T}$ containing $a$ zeros and $b$ ones where

$$
P_{e}(a, b) \triangleq \int_{0,1} \frac{1}{\pi \sqrt{(1-\theta) \theta}}(1-\theta)^{a} \theta^{b} d \theta
$$

This distribution, which allows sequential updating, i.e., $P_{e}(0,0)=1$, and for $a \geq 0$ and $b \geq 0$

$$
P_{e}(a+1, b)=\frac{a+\frac{1}{2}}{a+b+1} \cdot P_{e}(a, b)
$$

Manuscript received October 16, 1994; revised February 22, 1996. The material in this correspondence was presented in part at the 14th Symposium on Information Theory in the Benelux, Veldhoven, The Netherlands, May 17-18, 1993.

F. M. J. Willems and T. J. Tjalkens are with the Electrical Engineering Department, Eindhoven University of Technology, P.O. Box 513, $5600 \mathrm{MB}$ Eindhoven, The Netherlands.

Y. M. Shtarkov is with the Institute for Problems of Information Transmission, 101447, Moscow, GSP-4, Russia.

Publisher Item Identifier S 0018-9448(96)06056-7.

${ }^{1}$ The notation of [14] is used. and

$$
P_{e}(a, b+1)=\frac{b+\frac{1}{2}}{a+b+1} \cdot P_{e}(a, b)
$$

was suggested by Krichevsky and Trofimov [3]. Its parameter redundancy can be uniformly bounded, i.e., for any sequence $x_{1}^{T}$ with actual probability $P_{a}\left(x_{1}^{T}\right)=(1-\theta)^{a} \theta^{b}$, it can be shown (see [14] and note that $T \neq 0$ ) that

$$
\log \frac{P_{\alpha}\left(x_{1}^{T}\right)}{P_{c}\left(x_{1}^{T}\right)} \leq \frac{1}{2} \log T+1, \quad \text { for all } \theta \in[0,1] .
$$

In a more general setting, the source is not memoryless. The distribution that the source uses to generate the next symbol $X_{t}, t=$ $1, \cdots, T$, is determined by the binary sequence $u_{t}(1), \cdots, u_{t}(D)$ of length $D$, which is called the context of $x_{t}$. One can think of sources for which the context consists of the $D$ most recent source outputs, thus $u_{t}(d)=x_{t-d}$, for $d=1, \cdots, D$. More general context definitions are possible; it is assumed, however, that the context $u_{t}(1), \cdots, u_{t}(D)$ is always available to the encoder at the encoding time of $x_{t}$ and to the decoder at the decoding time of $x_{t}$.

The mapping $M$ from the context space $\{0,1\}^{D}$ into the parameter-index set $\mathcal{K}$, is what we call the model of the source. To each parameter-index $k \in \mathcal{K}$ there corresponds a parameter $\theta_{k} \in[0,1]$. The source generates $X_{t}$, with a probability of a 1 equal to $\theta_{M\left(u_{t}(1), \cdots, u_{t}(D)\right)}$. It should be noted that some authors (e.g., Rissanen) use the word model to indicate a source (a probability distribution). However, in our setting the word model stands for the structure of a source, i.e., the mechanism that enables a certain parameter given a context.

If the actual source has model $M_{a}$, and we know this model, we can partition the sequence $x_{1}^{T}$ in memoryless subsequences and use

$$
P_{c}\left(x_{1}^{T} \mid M_{a}\right)=\prod_{k \in \mathcal{K}_{a}} P_{e}\left(a_{k}, b_{k}\right)
$$

as a coding distribution, where $a_{k}$ (resp., $\left.b_{k}\right)$ is the number of instants $t$ for which $x_{t}=0$ (resp., 1) and $M_{a}\left(u_{t}(1), \cdots, u_{t}(D)\right)=k$. The image of $\{0,1\}^{D}$ under $M_{a}$ is $\mathcal{K}_{a}$. Again this coding distribution allows sequential updating. For any sequence $x_{1}^{T}$, using (4) and the convexity of the $\log (\cdot)$, the parameter redundancy can now be upper-bounded as

$$
\begin{aligned}
& \log \frac{P_{a}\left(x_{1}^{T}\right)}{P_{c}\left(x_{1}^{T} \mid M_{a}\right)}=\sum_{k \in \mathcal{K}_{a}} \log \frac{\left(1-\theta_{k}\right)^{a_{k}} \theta_{k}^{b_{k}}}{P_{e}\left(a_{k}, b_{k}\right)} \\
& \leq \sum_{k \in \mathcal{K}_{a}: a_{k}+b_{k}>0}\left(\frac{1}{2} \log \left(a_{k}+b_{k}\right)+1\right) \\
&=\left|\mathcal{K}_{a}\right| \sum_{k \in \mathcal{K}_{a}} \frac{1}{\left|\mathcal{K}_{a}\right|} \gamma\left(a_{k}+b_{k}\right) \\
& \leq\left|\mathcal{K}_{a}\right| \gamma\left(\sum_{k \in \mathcal{K}_{a}} \frac{a_{k}+b_{k}}{\left|\mathcal{K}_{a}\right|}\right) \\
&=\left|\mathcal{K}_{a}\right| \gamma\left(\frac{T}{\left|\mathcal{K}_{a}\right|}\right), \\
& \text { for } \theta_{k} \in[0,1], k \in \mathcal{K}_{a}
\end{aligned}
$$

where

$$
P_{a}\left(x_{1}^{T}\right)=\prod_{k \in \mathcal{K}_{a}}\left(1-\theta_{k}\right)^{a_{k}} \theta_{k}^{b_{k}}
$$


is the actual probability of $x_{1}^{T}$ and $\gamma(z) \triangleq z$ for $0 \leq z<1$ and $(1 / 2) \log (z)+1$. for $z \geq 1$. The function $\gamma(\cdot)$ is the smallest convex- $\cap$ continuation of $(1 / 2) \log (z)+1$ for $0 \leq z<1$ satisfying $\gamma(0)=0$.

Now suppose that the actual model $M_{a}$ belongs to a certain model class $\mathcal{M}$. If $M_{a}$ is unknown to the encoder and the decoder, we can weight the coding distributions corresponding to all models $M$ in the model class $\mathcal{M}$ and obtain coding distribution

$$
P_{c}\left(x_{1}^{T}\right)=\sum_{M \in \mathcal{M}} P_{\mathcal{M}}(M) P_{c}\left(x_{1}^{T} \mid M\right) .
$$

Here $P_{\mathcal{M}}(M)$ is the a priori probability that is assigned to the model $M$ in class $\mathcal{M}$. Of course

$$
\sum_{M \in \mathcal{M}} P_{\mathcal{M}}(M)=1
$$

We should be aware of the fact that a model $M$ may have positive probability in one model class but zero probability in an other class. The reason for this is that each model class contains only certain models (structures). For any sequence $x_{1}^{T}$ the model redundancy relative to the corresponding model class $\mathcal{M}$ can now be upperbounded as

$$
\log \frac{P_{c}\left(x_{1}^{T} \mid M_{a}\right)}{P_{c}\left(x_{1}^{T}\right)} \leq \log \frac{1}{P_{\mathcal{M}}\left(M_{a}\right)}, \quad \text { for all } M_{a} \in \mathcal{M}
$$

simply by using

$$
P_{c}\left(x_{1}^{T}\right)=\sum_{M \in \mathcal{M}} P_{\mathcal{M}}(M) P_{c}\left(x_{1}^{T} \mid M\right) \geq P_{\mathcal{M}}\left(M_{a}\right) P_{c}\left(x_{1}^{T} \mid M_{a}\right)
$$

The total cumulative redundancy corresponding to the weighted coding distribution $P_{c}\left(x_{1}^{T}\right)$ is now equal to the sum of the (cumulative) model, parameter and coding redundancies. Using (1), (5), and (6) we can upper-bound this total redundancy for any sequence $x_{1}^{T}$ in the following way:

$$
\begin{aligned}
L\left(x_{1}^{T}\right)-\log \frac{1}{P_{a}\left(x_{1}^{T}\right)}= & \log \frac{P_{c}\left(x_{1}^{T} \mid M_{a}\right)}{P_{c}\left(x_{1}^{T}\right)}+\log \frac{P_{a}\left(x_{1}^{T}\right)}{P_{c}\left(x_{1}^{T} \mid M_{a}\right)} \\
& +L\left(x_{1}^{T}\right)-\log \frac{1}{P_{c}\left(x_{1}^{T}\right)} \\
< & \log \frac{1}{P_{\mathcal{M}}\left(M_{a}\right)}+\left|\mathcal{K}_{a}\right| \gamma\left(\frac{T}{\left|\mathcal{K}_{a}\right|}\right)+2 .
\end{aligned}
$$

This holds for all models $M_{a} \in \mathcal{M}$ and parameters $\theta_{k} \in[0,1]$, $k \in \mathcal{K}_{a}$.

Rewriting this bound, and taking the minimum over all source models $M_{a}$ and corresponding parameters we obtain for all $x_{1}^{T}$ (not necessarily being generated by a source having an actual model that belongs to $\mathcal{M}$ )

$$
\begin{aligned}
L\left(x_{1}^{T}\right)<\min _{M_{a} \in \mathcal{M}, \theta_{k} \in[0,1], k \in \mathcal{K}_{a}\{}\{ & \log \frac{1}{P_{a}\left(x_{1}^{T}\right)}+\log \frac{1}{P_{\mathcal{M}}\left(M_{a}\right)} \\
& \left.+\left|\mathcal{K}_{a}\right| \gamma\left(\frac{T}{\left|\mathcal{K}_{a}\right|}\right)+2\right\} .
\end{aligned}
$$

From this we may conclude that context weighting methods try to minimize the total description length of the sequence $x_{1}^{T}$, relative to a given model class. So we can say that we fit all the sources with models in the given class to the sequence. The codeword length is determined by the best fitting source in the sense of (8).

In the next sections we consider four different model classes. We show that for each of these four classes there exist natural a priori distributions over the models in the class. These distributions allow efficient (sequential) computation of the corresponding weighted probability $P_{c}\left(x_{1}^{T^{T}}\right)$.
It should be emphasized that model weighting is not a new idea. Ryabko's twice universal code [7] is very close to the method we suggest here. Also in Weinberger et al. [11] model weighting methods (these authors call it the double mixture techniques) are studied. It was our contribution, however, to demonstrate that for the class of tree sources, model weighting can be implemented in an efficient recursive way [14]. In this correspondence we show that this recursive contexttree weighting method can be generalized to classes of models that have a more complex structure.

\section{SplittingS}

It is natural to view a model as a partition of the set of all contexts $\{0,1\}^{D}$ into $|\mathcal{K}|$ cells (equivalence classes), one for each parameter $\theta_{k}, k \in \mathcal{K}$. Since each partition can be generated by a sequence of splittings, where each splitting is a partition into two cells, we assume that a model partitions subsets of $\{0,1\}^{D}$ into smaller subsets, performing splittings only. The model class determines which splittings are allowed, and therefore what the structure of the resulting context sets is and thus which models (structures) belong to the model class. A splitting which is always possible is the void splitting, corresponding to the assumption that all contexts in the considered subset are mapped onto the same parameter. Then, further splitting is unnecessary. Assuming that all possible splittings are equally likely, we can define a code that specifies a model in the class. This code is defined recursively, starting from the set of all contexts $\{0,1\}^{D}$. For each set of contexts the code is the concatenation of the code that specifies the splitting, followed by the two codes for the subsets that have resulted from the splitting, however, only when the splitting was nonvoid. The code of a singleton set, i.e., a set that contains only a single context, is always empty.

Example: Consider the case where $D=3$. We assume for our example source that it generates a new symbol according to parameter $\theta=0.8$ if the context $u(1) u(2) u(3)=000,001,010,101$, or 110 and according to parameter $\theta=0.1$ for contexts 011,100 , and 111 . If we allow arbitrary splitting (this corresponds to Model Class I as we will see soon) there are 127 possible splittings of eight contexts plus the void splitting. Therefore, we need $\log 128=7$ bits to specify this first splitting. After this splitting there are two context sets $\{000,001,010,101,110\}$ and $\{011,100,111\}$ we have to deal with. The code for each of these subsets can be the code for the void splitting since all contexts in these subsets correspond to the same parameter. To specify this void splitting we need 4 bits for the first subset and 2 bits for the second one. In total 13 bits are needed to describe the model $M$ that matches best (i.e., with the lowest possible number of parameters) to our example source if arbitrary splitting is allowed, resulting in $|\mathcal{K}|=2$ parameter indices.

In the next section we will see that these splittings lead to efficient weighting methods.

\section{MOdel ClaSSES}

Weighting is assigning probabilities to subsequences corresponding to context subsets. The subsequence corresponding to a subset $\mathcal{S}$ of the set of all contexts $\{0,1\}^{D}$ is the concatenation of all source symbols $x_{t}$ with contexts $u_{t}(1), \cdots, u_{t}(D)$ in $\mathcal{S}$. The problem now is whether this subsequence should be considered memoryless or whether the context set $\mathcal{S}$ (and also the subsequence) should be further split. For a memoryless subsequence we can use the estimator $P_{e}(\mathcal{S}) \triangleq P_{e}\left(a_{S}, b_{\mathcal{S}}\right)$ where $a_{S}\left(\right.$ resp., $\left.b_{S}\right)$ is the number of instants $t$ for which $u_{t}(1), \cdots, u_{t}(D) \in \mathcal{S}$ and $x_{t}=0$ (resp., 1). If splitting of $\mathcal{S}$ into $\mathcal{S}_{1}$ and $\mathcal{S}_{2}$ is necessary, we should multiply the weighted probabilities for the subsequences corresponding to the subsets $\mathcal{S}_{1}$ and $\mathcal{S}_{2}$ that result from the splitting, i.e., $P_{w}\left(\mathcal{S}_{1}\right)$ and $P_{w}\left(\mathcal{S}_{2}\right)$, with 


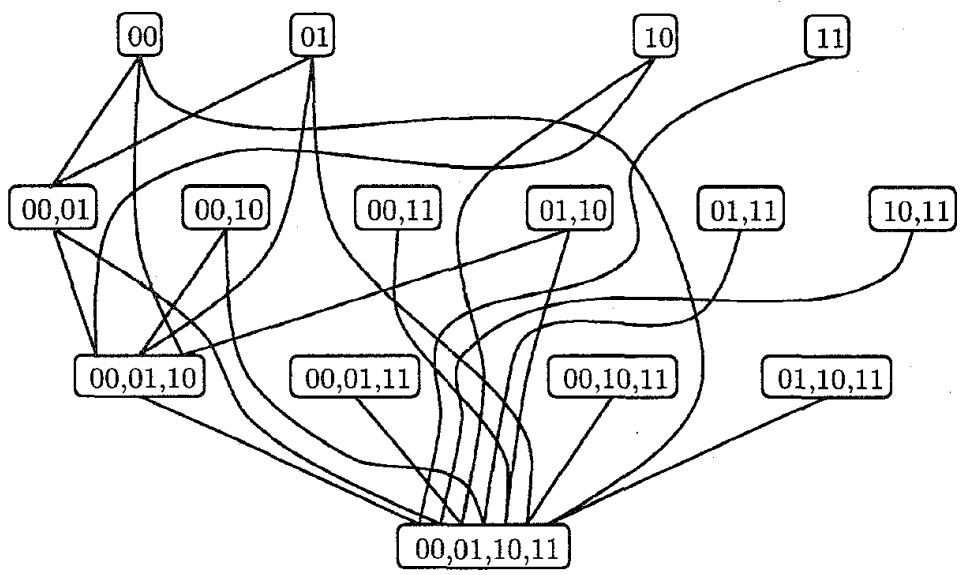

Fig. 1. Arbitrary splitting graph for $D=2$. Only the splittings of $\{00,01,10,11\},\{00,01,10\}$, and $\{00,01\}$ are shown.

each other. Since none of the alternatives is more favorable than the others, we just average the weighted probabilities corresponding to all the splittings, including the (estimated) probability of the void splitting. The weighted probability of a singleton set, i.e., a set with that contains only one context, is always its estimated probability, i.e.,

$$
P_{w}(\mathcal{S}) \triangleq P_{e}(\mathcal{S}), \quad \text { if }|\mathcal{S}|=1 .
$$

\section{A. Class I: Arbitrary Splitting}

Let $\Pi(\mathcal{S})$ be the set of all (nonvoid) splittings of $\mathcal{S}$, i.e.,

$$
\begin{aligned}
& \Pi(\mathcal{S}) \triangleq\left\{\left\{\mathcal{S}_{1}, \mathcal{S}_{2}\right\} \mid \mathcal{S}_{1}\right. \neq \phi, \mathcal{S}_{2} \neq \phi \\
&\left.\mathcal{S}_{1} \cap \mathcal{S}_{2}=\phi, \mathcal{S}_{1} \cup \mathcal{S}_{2}=\mathcal{S}\right\} .
\end{aligned}
$$

Note that the number of splittings of $\mathcal{S}$ including the void splitting is in this case equal to $2^{|\mathcal{S}|-1}$. The recursive weighting algorithm for this most general form of splitting is now defined as (see also Fig. 1)

$$
P_{w}(\mathcal{S}) \triangleq \frac{P_{\mathrm{e}}(\mathcal{S})+\sum_{\left\{\mathcal{S}_{1}, \mathcal{S}_{2}\right\} \in \Pi(\mathcal{S})} P_{w}\left(\mathcal{S}_{1}\right) P_{w}\left(\mathcal{S}_{2}\right)}{2^{|\mathcal{S}|-1}}
$$

for $|\mathcal{S}|>1$. For $\mathcal{S}$ such that $|\mathcal{S}|=1$ we take $P_{w}(\mathcal{S}) \triangleq P_{e}(\mathcal{S})$. The weighted probability $P_{w}\left(\{0,1\}^{D}\right)$ can now be used as coding probability. What we mean by this is the following. Suppose data (context and source symbols) are processed in a structure of records, one for each subset $\mathcal{S}$ of $\{0,1\}^{D}$, for $1, \cdots, t-1$. The record corresponding to set $\mathcal{S}$ contains $a_{\mathcal{S}}, b_{\mathcal{S}}, P_{e}(\mathcal{S})$, and $P_{w}(\mathcal{S})$. Then

$$
P_{c}\left(x_{1}^{t-1}\right)=P_{w}\left(\{0,1\}^{D}\right) \text {. }
$$

Now the context $u_{t}(1), \cdots, u_{t}(D)$ becomes available to encoder and decoder. Updating the structure of records with $X_{t}=0$ would yield the block probability $P_{c}\left(x_{1}^{t-1}, X_{t}=0\right)$ in the root of the structure and updating with $X_{t}=1$ gives $P_{c}\left(x_{1}^{t-1}, X_{t}=1\right)$ in the root. Updating the structure means that all records corresponding to $\mathcal{S}$ such that $u_{t}(1), \cdots, u_{t}(D) \in \mathcal{S}$ should be updated. Updating of a record $\mathcal{S}$ is done by multiplying $P_{e}(\mathcal{S})$ by either

$$
\left(a_{\mathcal{S}}+1 / 2\right) /\left(a_{\mathcal{S}}+b_{\mathcal{S}}+1\right), \quad \text { if } X_{t}=0
$$

or by

$$
\left(b_{\mathcal{S}}+1 / 2\right) /\left(a_{\mathcal{S}}+b_{\mathcal{S}}+1\right), \quad \text { if } X_{t}=1
$$

incrementing $a_{\mathcal{S}}$ by one if $X_{t}=0$ or $b_{\mathcal{S}}$ by one if $X_{t}=1$, and updating $P_{w}(\mathcal{S})$, which can only be done if the weighted probabilities of the split versions of $\mathcal{S}$ that contain $u_{t}(1), \cdots, u_{t}(D)$ have already been updated. The dummy probabilities in the root of the structure (you do not actually update the structure) can now be used for sequential encoding and decoding. It is easily checked that

$$
P_{c}\left(x_{1}^{t-1}, X_{t}=0\right)+P_{c}\left(x_{1}^{t-1}, X_{t}=1\right)=P_{c}\left(x_{1}^{t-1}\right) \text {. }
$$

After encoding or decoding the actual update is performed with the actual value of $X_{t}$.

The total number of records in the structure can be as large as $2^{2^{D}}-1$. For each source symbol $2^{2^{D}-1}$ records must be updated.

Inspection shows that the models are weighted with an a priori distribution which is equal to the sum of the probabilities induced by the lengths of all codes that specify the model; i.e., the partition of $\{0,1\}^{D}$.

Example (Continued): The model in Class I that matches best to our example source is given by

$$
M(000)=M(001)=M(010)=M(101)=M(110)=0
$$

and

$$
M(011)=M(100)=M(111)=1 .
$$

It has $|\mathcal{K}|=2$ parameters $\left(\theta_{0}\right.$ and $\left.\theta_{1}\right)$. The model redundancy of our example source is therefore at most 13 bits. There is only one sequence of splittings that specifies the model $M$ in Class $I$. Therefore

$$
\begin{aligned}
P_{w}\left(\{0,1\}^{3}\right) \geq & 2^{-7} P_{w}(\{000,001,010,101,110\}) \\
& \cdot P_{w}(\{011,100,111\}) \\
\geq & 2^{-7} 2^{-4} P_{e}(\{000,001,010,101,110\}) 2^{-2} \\
& \cdot P_{e}(\{011,100,111\}) \\
= & 2^{-13} P_{e}(\{000,001,010,101,110\}) \\
& \cdot P_{e}(\{011,100,111\}) .
\end{aligned}
$$

Note that in general there are several sequences of splittings that specify a model in Model Class I.

\section{B. Class II: Lexicographical Splitting}

Define

$$
B\left(u_{1}, \cdots, u_{D}\right) \triangleq \sum_{d=1, D} u_{d} 2^{D-d}
$$

as the index of the context $u_{1}, \cdots, u_{D}$. This index determines a lexicographical ordering over the set of contexts. For $0 \leq i<j \leq 2^{D}$ 


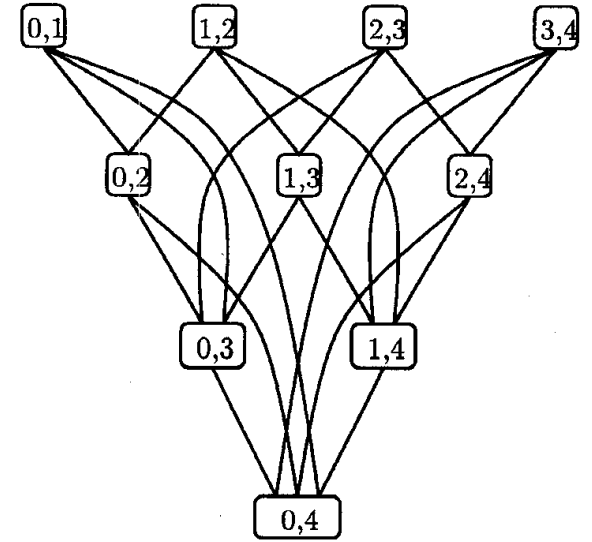

Fig. 2. Lexicographical splitting graph for $D=2$.

define $\mathcal{S}_{i, j}$ as the set of all contexts with an index between $i$ and $j$, i.e.,

$$
\mathcal{S}_{i, j} \triangleq\left\{s \in\{0,1\}^{D} \mid i \leq B(s)<j\right\} .
$$

The recursive weighting procedure for lexicographical splitting is defined by (see also Fig. 2)

$$
P_{w}\left(\mathcal{S}_{i, j}\right) \triangleq \frac{P_{e}\left(\mathcal{S}_{i, j}\right)+\sum_{k=i+1, j-1} P_{w}\left(\mathcal{S}_{i, k}\right) P_{w}\left(\mathcal{S}_{k, j}\right)}{j-i}
$$

for $j-i>1$. For sets $\mathcal{S}_{i, j}$ with $j-i=1$ we take

$$
P_{w}\left(\mathcal{S}_{i, j}\right) \triangleq P_{e}\left(\mathcal{S}_{i, j}\right) \text {. }
$$

Probability

$$
P_{w}\left(S_{0,2^{D}}\right)=P_{w}\left(\{0,1\}^{D}\right)
$$

can be used for sequential encoding and decoding.

The total number of records in the structure can now be as large as $2^{D-1}\left(2^{D}+1\right)$. For a source symbol that occurs with a context with index $i$ there are $(1+i)\left(2^{D}-i\right)$ records that need updating. This is never more than $2^{D-1}\left(2^{D-1}+1\right)$.

Example (Continued): The model $M$ in Class II that matches best to the example source has

and

$$
\begin{aligned}
M(000)=M(001) & =M(010)=0 \\
M(011) & =M(100)=1 \\
M(101) & =M(110)=2
\end{aligned}
$$

$$
M(111)=3
$$

and hence $|\mathcal{K}|=4$ parameters (however, with $\theta_{0}=\theta_{2}$ and $\theta_{1}=\theta_{3}$ ).

The model redundancy of our example source is at now most 8.599 bits. This follows from the decomposition

$$
\begin{aligned}
P_{w}\left(\mathcal{S}_{0,8}\right) \geq & \frac{1}{8} P_{w}\left(\mathcal{S}_{0,3}\right) P_{w}\left(\mathcal{S}_{3,8}\right)+\cdots \\
\geq & \frac{1}{8} \frac{1}{3} P_{e}\left(\mathcal{S}_{0,3}\right) \frac{1}{5} P_{w}\left(\mathcal{S}_{3,5}\right) P_{w}\left(\mathcal{S}_{5,8}\right)+\cdots \\
\geq & \frac{1}{8} \frac{1}{3} P_{e}\left(\mathcal{S}_{0,3}\right) \frac{1}{5} \frac{1}{2} P_{e}\left(\mathcal{S}_{3,5}\right) \\
& \quad \cdot \frac{1}{3} P_{w}\left(\mathcal{S}_{5,7}\right) P_{w}\left(\mathcal{S}_{7,8}\right)+\cdots
\end{aligned}
$$

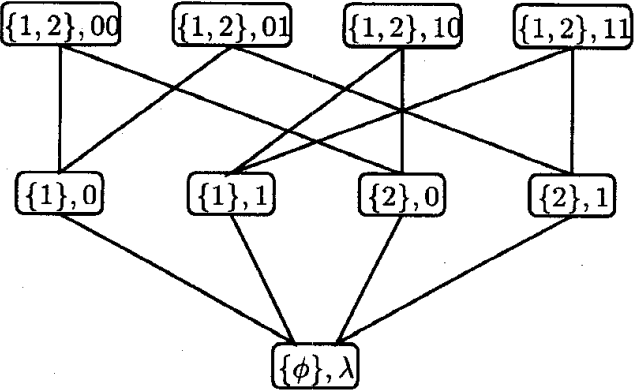

Fig. 3. Arbitrary position splitting graph for $D=2$.

$$
\begin{aligned}
\geq & \frac{1}{8} \frac{1}{3} P_{e}\left(\mathcal{S}_{0,3}\right) \frac{1}{5} \frac{1}{2} P_{e}\left(\mathcal{S}_{3,5}\right) \\
& \quad \cdot \frac{1}{3} \frac{1}{2} P_{e}\left(\mathcal{S}_{5,7}\right) \frac{1}{1} P_{e}\left(\mathcal{S}_{7,8}\right)+\cdots \\
= & \frac{1}{1440} P_{e}\left(\mathcal{S}_{0,3}\right) P_{e}\left(\mathcal{S}_{3,5}\right) P_{e}\left(\mathcal{S}_{5,7}\right) P_{e}\left(\mathcal{S}_{7,8}\right)+\cdots \\
= & \frac{13}{5040} P_{e}\left(\mathcal{S}_{0,3}\right) P_{e}\left(\mathcal{S}_{3,5}\right) P_{e}\left(\mathcal{S}_{5,7}\right) P_{e}\left(\mathcal{S}_{7,8}\right) .
\end{aligned}
$$

There are five sequences of splittings that specify the best model $M$ in Class II. One of these is

$$
\mathcal{S}_{0,8} \rightsquigarrow \mathcal{S}_{0,3} \mathcal{S}_{3,8} \rightsquigarrow \mathcal{S}_{0,3} \mathcal{S}_{3,5} \mathcal{S}_{7,8} \rightsquigarrow \mathcal{S}_{0,3} \mathcal{S}_{3,5} \mathcal{S}_{5,7} \mathcal{S}_{7,8} .
$$

The length of the specification code for this splitting is

$$
\begin{array}{r}
\log 8+(\log 3)+(\log 5+(\log 2)+(\log 3+(\log 2)+(\log 1))) \\
=\log 1440=10.492
\end{array}
$$

bits. The induced probability of this splitting is $1 / 1440$. Together the five sequences of splittings contribute $13 / 5040$ to the model $M$. Note that the number of parameters of the best model for our example source in Class II is higher than in Class I. This leads to a higher parameter redundancy for lexicographical splitting than for arbitrary splitting. Note that we have seen that in Class II there is only one best model for our example source which, however, can be reached by several splitting sequences.

\section{Class III: Arbitrary Position Splitting}

In this class (and also in Class IV) context subsets are split according to the value of a context digit. The position of this context digit can be arbitrary, i.e., in $\{1, \cdots, D\}$ in Class III. A subset is determined by the set $\mathcal{P}$ of positions and the sequence of values

$$
\prod_{d \in \mathcal{P}} v_{d}
$$

at these positions, hence,

$$
\mathcal{S}_{\mathcal{P}, \prod_{d \in \mathcal{P}} v_{d}} \triangleq\left\{u_{1}, \cdots, u_{D} \mid u_{d}=v_{d}, d \in \mathcal{P}\right\} .
$$

Recursive weighting for arbitrary position splitting is defined by (15) at the bottom of this page (see Fig. 3) for position sets $\mathcal{P} \neq$ $\{1, \cdots, D\}$. For subsets containing a single context, i.e., subsets for which all positions are specified, we have

$$
\begin{aligned}
P_{w}\left(\mathcal{S}_{\{1, \cdots, D\}, v_{\mathbf{1}} \cdots v_{D}}\right) & =P_{e}\left(\mathcal{S}_{\{1, \cdots, D\}, v_{\mathbf{1}}, \cdots, v_{D}}\right) \\
& =P_{E}\left(\left\{v_{1}, \cdots, v_{D}\right\}\right) .
\end{aligned}
$$

The weighted probability $\left.P_{w}\left(S_{\{\phi\}, \lambda}\right)=P_{w}\left(\{0,1\}^{D}\right\}\right)$ can be used for sequential encoding and decoding. Here $\{\phi\}$ is the empty set, and $\lambda$ the empty sequence.

$$
P_{w}\left(\mathcal{S}_{\mathcal{P}, \prod_{d \in \mathcal{P}} v_{d}}\right) \triangleq \frac{P_{e}\left(\mathcal{S}_{\mathcal{P}, \prod_{d \in \mathcal{P}} v_{d}}\right)+\sum_{p \in\{1, \ldots, D\}, p \notin \mathcal{P}} P_{w}\left(\mathcal{S}_{\mathcal{P} \cup\{\mathcal{P}\},\left(\prod_{d \in \mathcal{P}} v_{d}\right) \times 0}\right) P_{w}\left(\mathcal{S}_{\mathcal{P} \cup\{p\},\left(\prod_{d \in \mathcal{P}} v_{d}\right) \times 1}\right)}{D-|\mathcal{P}|+1}
$$


The total number of records in the structure can now be as large as $3^{D}$. For each source symbol $2^{D}$ records need to be updated.

Example (Continued): In this class there are no splitting sequences that lead to a model for our example source with less than five parameters. However, there are four splitting sequences that yield a model which matches our example source with five parameters. One of these sequences starts with a split on position 2 , resulting in a 0 branch and a 1 branch. The 0 branch is then split by position 1 , resulting in a 0 branch that is not split further and a 1 branch which is split by position 3 . The 1 branch resulting from the (first) split on position 2 is split by position 3 , resulting in a 0 branch and a 1 branch that are both not further split. The model that corresponds to this sequence can be described as

$$
\begin{aligned}
M(000)=M(001) & =0 \\
M(100) & =1 \\
M(101) & =2 \\
M(010)=M(110) & =3
\end{aligned}
$$

and

$$
M(011)=M(111)=4
$$

and has $|\mathcal{K}|=5$ (however, now $\theta_{0}=\theta_{2}=\theta_{3}$, and $\theta_{1}=\theta_{4}$ ). We obtain for the coding probability

$$
\begin{aligned}
P_{w}\left(\mathcal{S}_{\{\phi\}, \lambda}\right) \geq & \frac{1}{4} P_{w}\left(\mathcal{S}_{\{2\}, 0}\right) P_{w}\left(\mathcal{S}_{\{2\}, 1}\right)+\cdots \\
\geq & \frac{1}{4} \frac{1}{3} P_{w}\left(\mathcal{S}_{\{2,1\}, 00}\right) P_{w}\left(\mathcal{S}_{\{2,1\}, 01}\right) \\
& \cdot \frac{1}{3} P_{w}\left(\mathcal{S}_{\{2,3\}, 10}\right) P_{w}\left(\mathcal{S}_{\{2,3\}, 11}\right)+\cdots \\
\geq & \frac{1}{4} \frac{1}{3} \frac{1}{2} P_{e}\left(\mathcal{S}_{\{2,1\}, 00}\right) \frac{1}{2} P_{e}\left(\mathcal{S}_{\{2,1,3\}, 010}\right) \\
& \cdot P_{e}\left(\mathcal{S}_{\{2,1,3\}, 011}\right) \frac{1}{3} \frac{1}{2} P_{e}\left(\mathcal{S}_{\{2,3\}, 10}\right) \\
& \cdot \frac{1}{2} P_{e}\left(\mathcal{S}_{\{2,3\}, 11}\right)+\cdots \\
= & \frac{1}{576} P_{e}\left(\mathcal{S}_{\{2,1\}, 00}\right) P_{e}\left(\mathcal{S}_{\{2,1,3\}, 010}\right) \\
& \cdot P_{e}\left(\mathcal{S}_{\{2,1,3\}, 011}\right) P_{e}\left(\mathcal{S}_{\{2,3\}, 10}\right) \\
& \cdot P_{e}\left(\mathcal{S}_{\{2,3\}, 11}\right)+\cdots
\end{aligned}
$$

where we have considered only one sequence (the sequence described earlier) of splittings leading to a model with five parameters. This would yield a model redundancy for this model of 9.170 bits. Since there are three other splitting sequences that yield models $M$ with $|\mathcal{K}|=5$, and since we are considering a weighting method, we could say that the model redundancy (induced by these four models together, all having a priori probability $1 / 576$ ) is not more than 7.170 bits. Note that we have observed that in Class III our example source can be described by several (best) models, i.e., models with five parameters. Some of these models can be reached by more than one sequence of splittings.

\section{Class IV: Next Position Splitting}

In Class IV context subsets are split according to the value of the "next" context digit. Subsets are determined by the number $\delta$ of already split positions, and the sequence of values $\prod_{d=1, \delta} v_{d}$ at these split positions, thus

$$
\mathcal{S}_{\delta, \prod_{i=1, \delta} v_{d}} \triangleq\left\{u_{1}, \cdots, u_{D} \mid u_{d}=v_{d}, d=1, \cdots, \delta\right\} .
$$

The recursive weighting procedure for next position splitting is given by (17) at the bottom of this page (see also Fig. 4) for $\delta=0$,

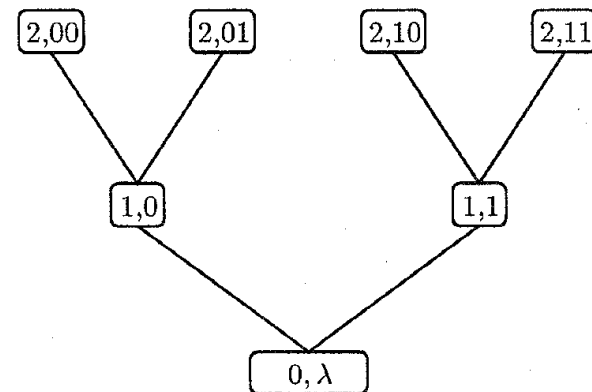

Fig. 4. Next position splitting graph (context tree) for $D=2$.

$1, \cdots, D-1$. For subsets containing a single context only, i.e., subsets for $\delta=D$, we have

$$
P_{w}\left(\mathcal{S}_{D}, v_{1}, \cdots, v_{D}\right)=P_{e}\left(\mathcal{S}_{D}, v_{1}, \cdots, v_{D}\right)=P_{e}\left(\left\{v_{1}, \cdots, v_{D}\right\}\right) .
$$

The weighted probability $P_{w}\left(S_{0, \lambda}\right)=P_{w}\left(\{0,1\}^{D}\right)$ can be used for sequential encoding and decoding.

The total number of records in the structure can now be as large as $2^{D+1}-1$. For each source symbol $D+1$ records need to be updated.

It should be noted that this model class is identical to the class of tree models defined in [12]. The weighting described in (17) is identical to context-tree weighting as described by the authors in [14].

Example (Continued): Our example source must now be modeled as follows:

$$
\begin{aligned}
M(000)=M(001) & =0 \\
M(010) & =1 \\
M(011) & =2 \\
M(100) & =3 \\
M(101) & =4 \\
M(110) & =5
\end{aligned}
$$

and

$$
M(111)=6
$$

and has $|\mathcal{K}|=7$ (however, now $\theta_{0}=\theta_{1}=\theta_{4}=\theta_{5}$ and $\theta_{2}=\theta_{3}=\theta_{6}$ ). The model redundancy is now 7 bits. We decompose as follows:

$$
\begin{aligned}
P_{w}\left(\mathcal{S}_{0, \lambda}\right) \geq & \frac{1}{2} P_{w}\left(\mathcal{S}_{1,0}\right) P_{w}\left(\mathcal{S}_{1,1}\right) \\
\geq & \frac{1}{2} \frac{1}{2} P_{w}\left(\mathcal{S}_{2,00}\right) P_{w}\left(\mathcal{S}_{2,01}\right) \frac{1}{2} P_{w}\left(\mathcal{S}_{2,10}\right) \\
& \cdot P_{w}\left(\mathcal{S}_{2,11}\right) \\
\geq & \frac{1}{2} \frac{1}{2} \frac{1}{2} P_{e}\left(\mathcal{S}_{2,00}\right) \frac{1}{2} P_{e}\left(\mathcal{S}_{3,010}\right) P_{e}\left(\mathcal{S}_{3,011}\right) \\
& \cdot \frac{1}{2} \frac{1}{2} P_{e}\left(\mathcal{S}_{3,100}\right) P_{e}\left(\mathcal{S}_{3,101}\right) \\
& \cdot \frac{1}{2} P_{e}\left(\mathcal{S}_{3,110}\right) P_{e}\left(\mathcal{S}_{3,111}\right) \\
= & \frac{1}{128} P_{e}\left(\mathcal{S}_{2,00}\right) P_{e}\left(\mathcal{S}_{3,010}\right) P_{e}\left(\mathcal{S}_{3,011}\right) \\
& \cdot P_{e}\left(\mathcal{S}_{3,100}\right) P_{e}\left(\mathcal{S}_{3,101}\right) \\
& \cdot P_{e}\left(\mathcal{S}_{3,110}\right) P_{e}\left(\mathcal{S}_{3,111}\right)
\end{aligned}
$$

In Class IV there is always only one model and sequence of splittings that specifies a source.

$$
\left.P_{w}\left(\delta, \mathcal{S}_{\prod_{d=1, \delta} v_{d}}\right) \triangleq \frac{P_{e}\left(\mathcal{S}_{\delta,}, \prod_{d=1, \delta} v_{d}\right)+P_{w}\left(\mathcal{S}_{\delta+1,\left(\prod_{d=1, \delta} v_{d}\right) \times 0}\right) P_{w}\left(\mathcal{S}_{\delta+1},\left(\prod_{d=1, \delta} v_{d}\right) \times 1\right.}{2}\right)
$$




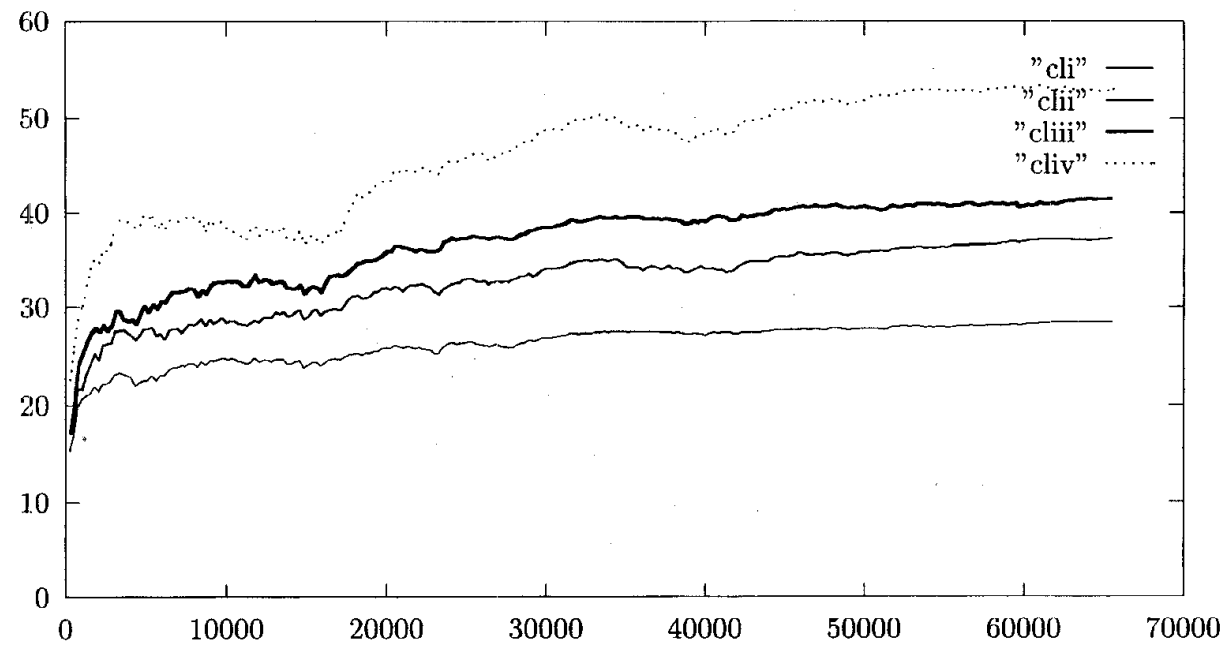

Fig. 5. Cumulative model plus parameter redundancies in bits for $t=1,2, \cdots, 2^{16}$.

\section{Simulations}

Example (Continued): We have simulated the example source such that it generates information according to context definition $u_{t}(d)=x_{t-d}, d=1, D$, with $D=3$. The source produced a sequence of $T=2^{16}$ binary digits (after having generated three digits that were necessary to form the first three contexts). We computed for this sequence for each of the four procedures defined by (11), (13), (15), and (17), for $t=1,2, \cdots, T$ the redundancy $\log P_{a}\left(x_{1}, \cdots, x_{t}\right) / P_{c}\left(x_{1}, \cdots, x_{t}\right)$, which is the total redundancy under the assumption that there is no coding redundancy. The results are plotted in Fig. 5 .

In the previous section we have seen that the model redundancies for our source in each of the four classes are upper-bounded by $13.000,8.599,7.170$, resp., 7.000 bits. Upper bound (5) leads to parameter redundancies that cannot exceed $17.000,32.000,39.195$, resp., 53.174 bits for our source in the four different classes. The total redundancies are therefore upper-bounded by $30.000,40.599,46.365$, resp., 60.174 bits. The figure shows that the computed redundancies are close to these bounds.

\section{REMARKS}

For a fixed model $M$, Rissanen's lower bound [5] implies that for any block code the expected redundancy per source symbol is not less than roughly $(|\mathcal{K}| / 2 T) \log T$ for all parameter vectors $\left(\theta_{1}, \theta_{2}, \cdots, \theta_{\mathcal{K}}\right)$ except a set of vectors whose measure goes to 0 as $T \rightarrow \infty$. Here $\mathcal{K}$ is the parameter-index set corresponding to $M$ and $|\mathcal{K}|$ the number of parameters. Earlier results of Krichevsky [2] for the memoryless case and Trofimov [9] for Markov sources state that for any code there is at least one parameter vector for which the expected redundancy is not smaller than $(|\mathcal{K}| / 2 T) \log T$ for large $T$. Furthermore, given model $M$ it follows from the work of Shtarkov [8] that for any code there exists a sequence $x_{1}^{T}$ and a parameter vector such that the individual redundancy with respect to the corresponding source is not less than $(|\mathcal{K}| / 2 T) \log T$ asymptotically. Recently, Weinberger et al. [11] showed that this lower bound holds for most sequences in most types. Our weighting methods achieve all these lower bounds in the sense that the redundancy per source symbol is not more than $(|\mathcal{K}| / 2 T) \log T$ for large $T$. Despite this it is not clear whether or not our weightings are optimal in a certain sense. Each weighting over the models in a model class determines cumulative (upper bounds (6) on the) model redundancies. Our weightings are induced by the probabilities that we have assigned to the splittings; in other words, the weightings are such that they lead to simple implementations. They do, however, have the pleasant property that the model redundancy increases when the model gets more complex. A criterion for optimality could be to minimize the (upper bound on the) model redundancy per parameter, under the constraint that the model redundancy is proportional to the number of parameters. For Class IV, this criterion leads to a weighting satisfying [14, relation (32)] with $-\log \alpha_{0}=\delta$. It is an open problem, however, to find similar solutions for the other three model classes.

Although we have only considered binary sources and binary contexts here, it is straightforward to generalize to nonbinary cases. In our presentation of the weighting algorithms we assume infinite precision arithmetic. Modifications exist, however, that can be implemented on fixed register-length machines.

Weinberger et al., in [10, Section V] considered a problem which is similar to the Class I case. They suggest to check whether it is beneficial to combine contexts and context sets (possibly while running a Class IV algorithm). These sets of contexts then determine the estimated next-symbol distribution (plug-in approach). The number of possible comparisons is of course very large. The method we propose here is an elegant way to weight all the alternatives systematically; however, this also can only be applied for small values of $D$, say $D \leq 4$.

If instead of having a good datacompression code we are interested in finding the model $M$ in model class $\mathcal{M}$ that minimizes (the description length)

$$
\log \frac{1}{\prod_{k \in \mathcal{K}(M)} P_{e}\left(a_{k}, b_{k}\right)}+\log \frac{1}{P_{\mathcal{M}}(M)}
$$

we can change the context weighting methods defined in (11), (13), (15), and (17), into context maximizing methods. Instead of adding up the probabilities corresponding to all the alternative splittings (including the void one) and dividing by the number of splittings in each node, we can take the maximum over all these probabilities and divide. Tracking this procedure yields the minimizing model. A similar procedure occurs in Nohre [15].

These context maximizing methods are closely related to classification procedures based on Rissanen's minimum description length principle (see [6] and also Quinlan and Rivest [4]). Considering the attributes, or tests, of an object $t$ as its context $u_{t}(1), \cdots, u_{t}(D)$ and the class of the object as source output $x_{t}$, classification can be regarded as a source-coding problem. 
The flexibility of context maximizing, allows us to describe efficient methods for producing minimum description length classification structures. Combinations of our maximizing methods for the different classes lead to interesting classification procedures, even for attributes that take values in "large" alphabets. Note that the algorithm for Class III selects the positions (attributes) which gives the highest reduction of the description length and produces a decision tree (see Quinlan and Rivest [4]), while Class II methods can be used to find the most effective thresholds in large attribute alphabets. The fact that there exist elegant context weighting methods to treat missing attributes demonstrates once more the flexibility of context weighting (maximizing).

\section{ACKNOWLEDGMENT}

The authors wish to thank the reviewers and the Associate Editor M. Feder for valuable comments.

\section{REFERENCES}

[1] F. Jelinek, Probabilistic Information Theory. New York: McGraw-Hill, 1968 , pp. $476-489$

[2] R. E. Krichevsky, "The connection between the redundancy and the reliability of information about the source," Probl. Inform. Transm., vol. 7, no. 3, pp. $48-57,1968$

[3] R. E. Krichevsky and V. K. Trofimov, "The performance of universal encoding," IEEE Trans. Inform. Theory, vol. IT-27, no. 2, pp. 199-207, Mar. 1981.

[4] J. R. Quinlan and R. L. Rivest, "Inferring decision trees using the minimum description length principle," Inform. Comput., vol. 80, pp. 227-248, 1989.

[5] J. Rissanen, "Universal coding, information, prediction, and estimation," IEEE Trans. Inform. Theory, vol. IT-30, no. 4, pp. 629-636, July 1984.

[6] _ Stochastic Complexity in Statistical Inquiry. Singapore: World Scientific, 1989.

[7] B. Ya. Ryabko, "Twice-universal coding," Probl. Inform. Transm., vol. 20, no. 3, pp. 24-28, 1984.

[8] Y. M. Shtarkov, "Methods of constructing lower bounds for redundancy of universal coding," Probl. Inform. Transm., vol. 18, no. 2, pp. 3-11, 1982.

[9] V. K. Trofimov, "The redundancy of universal encoding of arbitrary Markov sources," Probl. Inform. Transm., vol. 10, no. 4, pp. 16-24, 1974.

[10] M. J. Weinberger, A. Lempel, and J. Ziv, "A sequential algorithm for the universal coding of finite memory sources," IEEE Trans. Inform. Theory, vol. 38, no. 3, pp. 1002-1014, May 1992.

[11] M. J. Weinberger, N. Merhav, and M. Feder, "Optimal sequential probability assignment for individual sequences," IEEE Trans. Inform. Theory, vol. 40, no. 2, pp. 384-396, Mar. 1994.

[12] M. J. Weinberger, J. Rissanen, and M. Feder, "A universal finite memory source," IEEE Trans. Inform. Theory, vol. 41, no. 3, pp. 643-652, May 1995.

[13] F. M. J. Willems, Y. M. Shtarkov, and T. J. Tjalkens, "Context tree weighting: A sequential universal source coding procedure for FSMX sources," in IEEE Int. Symp. on Information Theory (San Antonio, TX, Jan. 17-22), 1993, p. 59.

[14] "The context-tree weighting method: Basic properties," IEEE Trans. Inform. Theory, vol. 41, no. 3, pp. 653-664, May 1995.

[15] R. Nohre, "Some topics in descriptive complexity," Ph.D. dissertation, Linköping Univ., Linköping, Sweden, 1998.

\section{Nearest Neighbor Decoding for Additive Non-Gaussian Noise Channels}

Amos Lapidoth, Member, IEEE

\begin{abstract}
We study the performance of a transmission scheme employing random Gaussian codebooks and nearest neighbor decoding over a power limited additive non-Gaussian noise channel. We show that the achievable rates depend on the noise distribution only via its power and thus coincide with the capacity region of a white Gaussian noise channel with signal and noise power equal to those of the original channel. The results are presented for single-user channels as well as multiple-access channels, and are extended to fading channels with side information at the receiver.
\end{abstract}

Index Terms - Nearest neighbor decoding, Euclidean distance, mismatched decoding, multiple-access channel, generalized cutoff rate, fading.

\section{INTRODUCTION}

Due to the simplicity of their implementation using matched filters and due to their robustness, nearest neighbor decoders (minimum Euclidean distance decoders) are often used on additive noise channels even if the noise is not a white Gaussian process. For such channels, the nearest neighbor decoding rule is usually suboptimal and a loss in performance is thus incurred. In this correspondence we quantify this loss in terms of the achievable rates, i.e., the rates at which reliable communication is possible with a nearest neighbor decoder.

Stated as above, the problem is a special case of the general mismatch problem that has been studied in [1]-[5] for the singleuser channel, and in [6] for the multiple-access channel. However, the techniques that we use in order to study the problem are quite different from those used in the above references and rely heavily on Euclidean geometry. This allows us to deal with the infinite input and output alphabets and with the memory that the noise may exhibit.

It should be noted that we only study the case where the transmitter uses random Gaussian codebooks. While this is the optimal input distribution for white Gaussian channels, this may not necessarily be optimal for non-Gaussian channels and the decoder that we assume, see Example 1.

The motivation to assume this input distribution is that Gaussian codebooks are relatively well understood, and that, as we shall see, Gaussian codebooks and nearest neighbor decoders form a very robust communication scheme. Furthermore, for the power-limited additive noise single-user channel, Gaussian noise and Gaussian signaling constitute a saddle-point for the mutual information functional [7]: given that the noise is Gaussian the input distribution that maximizes the mutual information between the channel input and output is the Gaussian distribution, and given that the input distribution is Gaussian, the worst noise, i.e., the noise that minimizes

Manuscript received May 9, 1995; revised Feb. 20, 1996. This work was supported in part by NSF under Grant NCR-9205663, JSEP under Contract DAAH04-94-G-0058, and ARPA under Contract J-FBI-94-218-2. The material in this correspondence was presented in part at the IEEE Inernational Symposium on Information Theory, Trondheim, Norway, June 1994.

The author is with the Department of Electrical Engineering and Computer Science, Massachusetts Institute of Technology, Cambridge, MA 02139-4307 USA.

Publisher Item Identifier S 0018-9448(96)05225-X. 